\title{
Research on the Novel and Efficient Mechanism of Exception Handling Techniques for Java
}

\author{
Xiaoqing $L v^{1}$ \\ ${ }^{1}$ huihua College Of Hebei Normal University, \\ Shijiazhuang,Hebei,050091 China
}

\begin{abstract}
With the development of software engineering and other computer science techniques, the application of Java based system is becoming popular. In this paper, we conduct theoretical analysis the novel and efficient mechanism of exception handling technique for Java. Not surprisingly, recurring failures and performance issues are often related to the poor exception handling actions implemented in real software systems. Exception handling mechanisms are meant to improve software robustness. Our proposed approach solves the issues well and effective.
\end{abstract}

Keywords: Java Programming; Exception Handling; Exception Testing; Novel Mechanism.

\section{INTRODUCTION}

Robust software systems must deliver their correct services and applications even in the presence of exceptions. An exception is an event that occurs during the normal execution of a software system or a general application, indicating that the system internal state is inconsistent. As the name suggests, the anomaly is a kind of unexpected circumstances, cause the program to break out of the running or deal with this situation to continue running. A typical example is the zero except the exception, at compile time is normal, but it will fail at run time, running and exit. To avoid program exits, must capture and handle exceptions. To write error-free programs is the biggest wish of every programmer, but the errors in the program is inevitable. It requires the programmer to design program must be capable of handling errors. In general, the error handling requirements are as follows: first, accurate detect errors, second for error processing, and the error conditions as far as possible to repair, let the program execution down as much as possible. Programming language of the anomaly occurred in the execution of the program of special events, would be a mistake which may also be a programmer to define a particular need to be aware of. This paper discusses the Java language exception handling technology, combined with the SUN's Java compiler to exception handling, puts forward using the Java language exception handling mechanism for software design, the correct way. Exception handling mechanism of programming language usually from the following several aspects: (1) Java exception handling mechanism of separated the exception handling code and common code, the granularity of reserve is block, rather than statements. Exception handling mechanism of the external manifestation of the Java language includes three parts. (2) The Java language exception handling mechanism of the static. Static exception types and captures its exception handler, the relationship between different abnormal matching different exception handlers which has nothing to do with the execution path. (3) Abnormal said. Java language use in the abnormal production and propagation of object representation is unusual, state information and can carry an exception occurs which is mainly the type of exception object. An exception occurs, the first to use the new create exception object in memory in the heap, then, to terminate the current execution paths, and release the exception object from the current environment of the handle. (4) Exception handling semantics, namely the invocation handler abnormal 
response after the transfer direction of program control flow. In the abnormal control theory, there are two basic methods: interrupt and recovery. The Java language using interrupt mode, where the exception happened after the termination of the executing, program process to exception handling and exception handling finished, returned to the calling point to continue, with no exception occurs and the contents of the normal program execution of the reserve [1-3].

To deal with the mentioned challenges, in this paper, we conduct corresponding research on the novel and efficient mechanism of exception handling technique for Java. Whenever Java program found during the operation of an identifiable operation error, namely the error when one exception class corresponding to it, the system will generate a corresponding the exception class object, generating an exception. SUN's Java compiler forced to check out the exception thrown to declare and the exception, and unrestricted to unchecked exceptions. No matter what kind of exception classes, once the exception object is produced, the Java exception handling mechanism will deal with it, to avoid the system crash, infinite loop or damage to the operating system. The detailed solution for the conditions is illustrated in the following sections.

\section{The Proposed Methodology}

The General Java Exception Handling Process. Compiler of exception handling, first by looking at compile time generated exception handling finish handle exception handling lookup table. In a Java virtual machine, including Java runtime data space stack, heap, method, constant pool area, Java is a method call stack instance, private, each Java virtual machine thread and thread at the same time to create the end at the same time. The Java stack method is used to store the corresponding operation state, including the operand stack, running environment, the local variable area, create the thread on the running environment contains a pointer to the method of components, an exception occurs, according to the pointer to find the appropriate method, each method in the Java exception handling table static associated with each method at compile time, exception handling, including the scope of its effective instruction in the table, can deal with the type of exception and exception handling code block address, this completes the exception handling handle lookup. Depending on the type of exception and effective instruction, if abnormal with the first catch block matching, was transferred to the catch block of code execution, after the execution, not empty if Finally pieces, execute Finally block, or directly out of the current method, has not been performed in the try block of statements and other catch block is ignored, equivalent to normally run ended. The method if the anomalies do not match with the first catch block, the system will automatically turn to the second catch block matching, if the second still does not match, turned to the third, until you find an acceptable catch block of the exception object. Abnormalities in inspection, therefore, the compiler enforcement method definition must declare all may throw exceptions, method calls, or to capture it, or spread exceptions to the upper method, even the Java virtual machine. The figure one shows the process.

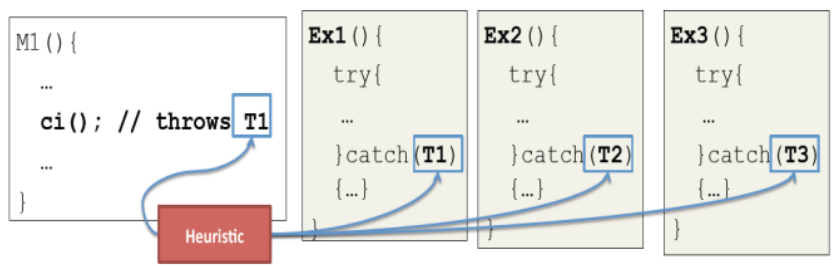

Fig. 1The General Java Exception Handling Process

Normally, checked exception must be treated, the programmer should set in view of the abnormal response might receive. First of all determine whether the caller has the ability to deal with it, if the caller can deal with it, or is unable to process at present, but the method called can handle, you can use check exception. If the caller to appear abnormal powerless, use unchecked exceptions, for example, to resource depletion when an exception is thrown, if the resource depletion is caused by excessive demand temporarily, belongs to the situation of 
recoverable, trigger a checked exception, depleted or program if it is really wrong, lead to unchecked exceptions. Checked and unchecked exceptions defined exceptions can be further divided into system and user-defined exception. System defined every exception class represents a run-time error class contains the run wrong information and the method of handling errors, etc. User-defined exceptions need programming personnel according to the special logic of the program in the user program create your own exception classes and the exception object, the operation of the corresponding specific to a particular application error. Java exceptions in the actual object, it is according to the hierarchical structure of the organization. In the following code block, we show the organization.

Java. Iang. ThrowabIe

+ - - java. Iang. Error

+ - java. Iang.

VirtuaImachineError

+ - - java. Iang. Exception

+ - - java. Iang.

IOException

RuntimeException

$$
+ \text { - java. Iang. }
$$

According to the characteristics of the procedure in the operation of the anomaly, the anomaly can be divided into synchronous and asynchronous anomaly in Java. In determining the program called synchronized anomaly, occurs not sure of the time and location of the Exception is called asynchronous Exception. Synchronous abnormal further divided into checked and unchecked exceptions, unchecked exceptions as a Runtime Exception or a subclass of Error and check the abnormal as Exception or Throwable subclass.

The Proposed Java Exception Process Steps. The design of our heuristics contemplated a set of structural facts extracted from source code because they are the easiest information related to exception handling to obtain. According to the Java virtual machine in the presence of the exception interrupt procedures of operation and to print out the abnormal information, namely the main treatment method is to record, from the Angle of the robustness, as far as possible to restore, general this kind of exception handling in the main program. Usually program of unchecked exceptions means not enough strong, if the program thoughtful, won't appear this kind of abnormal. Object-oriented inheritance is an important aspect of the class hierarchy, in a class define subclasses will inherit the characteristics of its parent class, and can have its own special qualities. Exception class hierarchy and object-oriented hierarchy and the alike, also has the inheritance, so software designers to along the exception hierarchy, exception handling on multiple levels. The following figure 2 shows the method called for the exception.

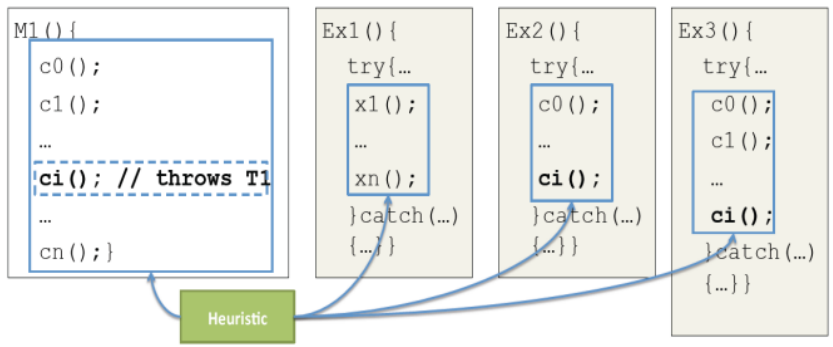

Fig. 2The General Methods Called for the Exception

In recovery mode, an exception is thrown, the control flow is transferred to the exception handler, the exception handler is responsible for correcting the operation was not completed, and then back to the location of the exception is thrown to continue. Invoke exception handlers in the pattern of the process and the normal procedure, the difference is performed after the exception handler to go back to the location of the exception is thrown, the location of the exception is thrown. Recovery mode determines the characteristics of the model is easy to implement, as a result, some exception handling structure adopted the recovery mode. To sum up, we observe that the exception mechanism processing pattern generally uses interrupt mode or recovery mode, but either model, with the exception handling code and alert. Alert zone represents a special code area, might have an anomaly in this area, followed by an exception handler in the alert area, if an exception is thrown when the running in the alert area code, then 
through exception propagation mechanism to find the corresponding exception handler.

The Access Control and Sample Code. Exception propagation mechanism to circulate the control to the exception handler, propagation mode is abandoned and the restoration of two types, corresponding processing pattern of interrupt and recovery mode. Abandon transmission means control flow will not be able to return to an exception is thrown, throw an exception point between the processor and the code is no longer be performed; Back propagation means that control flow will return to throw an exception, but can change the spread to abandon in the processor. Abandon spread and restoring the propagation mode can coexist in the same exception handling mechanism. Only at run time, must at run time to solve it, need software design personnel in view of such situation, and pass the relevant information in order to correctly deal with these problems in the operation process. In the statement or throw an exception when abnormal together with communication model, carried out in accordance with the different mechanisms, exception propagation mechanism refers to the way of looking for an exception handler, under normal circumstances, the dynamic transmission mechanism. The access control patter for the methodology is shown in the figure 3 .

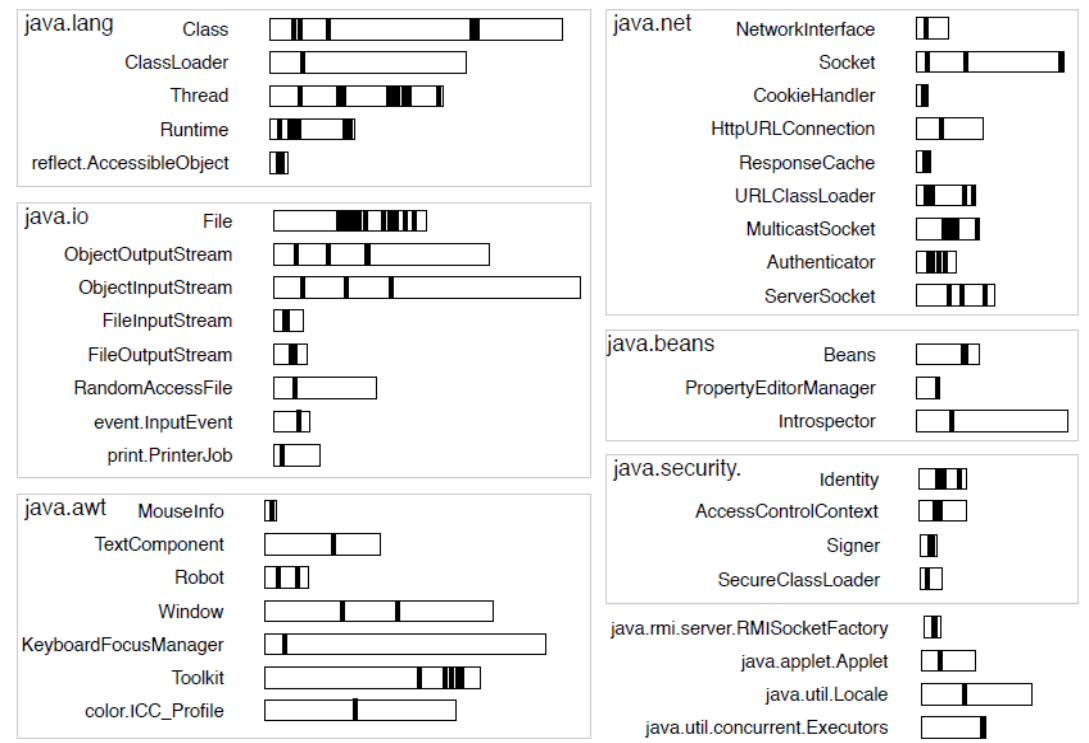

Fig. 3The Access Control Pattern for the Exception Process Steps

\section{CONCLUSIONS}

In this paper, we conduct corresponding research on the novel and efficient mechanism of exception handling technique for Java. Abnormity anomaly refers to the outside of the normal situation, does not mean that certain errors. Software system in abnormal very frequently, and even operating system, so we think very robust systems are also often appear abnormal. In the practical work, even if found and dealt with a lot of abnormal, in many cases the collapse of the system is still caused by abnormal. In order to enhance the robustness and reliability of the system, many programming languages introduced the exception handling mechanism. Our proposed approach combines the advanced access control model with the traditional model which will enhance the current pattern of designing the software. In the future, we plan to conduct more related research to polish and modify the current systematic approach. 


\section{Acknowledgement}

This research is supported by the project in Hebei province department of education (NO. 2012GJJG136). The Project name is: Introduce Companies to the Colleges-- The practice of innovation IT personnel training platform.

\section{References}

[1] Donna Malayeri, Aldrich J. Practical exception specifications[C]. //Lecture Notes in Computer Science. Springer-Verlag, 2006:200-220.

[2] Dony C, Knudsen J L, Romanovsky A, et al. Advanced Topics in Exception Handling Techniques[J]. Lecture Notes in Computer Science, 2006.

[3] Schröder L, Mossakowski T. Generic Exception Handling and the Java Monad.[J]. Algebraic Methodology and Software Technology, 2004:443-459. 